\title{
Characteristics of SSR Markers in Floccularia Luteo-virens by RNA -Sequencing
}

\author{
Kairui Lii, ${ }^{1,2}$, Ritian Qin ${ }^{1,2}$, Zhanling Xie ${ }^{1,2 *}$, Jing Guo ${ }^{1}$, Qing Meng ${ }^{1}$ and Hongyan Xu ${ }^{1}$ \\ ${ }^{1}$ National Key Laboratory Breeding Base for Innovation and Utilization of Plateau Crop Germplasm, China \\ ${ }^{2}$ College of Eco-Environmental Engineering, Qinghai University, China
}

Received: January 10, 2018; Published: January 18, 2018

*Corresponding author: Zhanling Xie, Key Laboratory Breeding Base for Innovation and Utilization of Plateau Crop Germplasm, No. 251, Ningda Road, Chengbei District, Xining, Qinghai Province, China, 810016, Email: xiezhanling2012@126.com

\begin{abstract}
Simple sequence repeats (SSRs) in Transcript and Unigene of Floccularia luteo-virens were determined by RNA sequencing. There were 4, 715 SSRs in 38, 988 Transcript sequences and 2, 910 SSRs in 22, 122 Unigene sequences using de novo sequencing, respectively. Mononucleotides showed the highest frequency of $26.16 \%$ in Transcript, and the ratio of mononucleotides was $\mathrm{A}>\mathrm{T}>\mathrm{G}>\mathrm{C}$; while in Unigene, dinucleotides showed the highest of $59.27 \%$, and the ratio of dinucleotides was TG/GT>AC/CA>AT/TA $>A G / G A$. The SSR with 10 repeats was the most abundant in Transcript (26.36\%), while that with six repeats was the most abundant in Unigene (22.71\%). Motif A had the highest frequency of $17.50 \%$ and $11.65 \%$ in Transcript and Unigene, while Motif C had the lowest frequency of $11.10 \%$ and $1.08 \%$ in Transcript and Unigene, respectively. For the PCR products, seven primers were screened and tested from the motifs, the result were in consistent with RNA sequencing results.
\end{abstract}

Keywords: Floccularia Luteo-virens; RNA-Sequencing; Characterization

Abbreviations: SSR: Simple Sequence Repeats

\section{Introduction}

Simple sequence repeats are short tandem repeats; SSRs are widespread in both coding and non-coding regions of prokaryotic and eukaryotic genomes with a high level of polymorphism [1]. Because of their high polymorphism, abundance, and co dominant inheritance, SSRs are well suited for the assessment of genetic diversity within crop species and the genetic relationships among species [2]. Floccularia luteo-virens, which is also referred to as yellow mushroom and belongs to Basidiomycotina, Hymenomycetes, Agaricales, Tricholomataceae, is known as one of the most appreciated wild mushrooms in China. The mushroom is an expensive edible medical fungus, rich in protein, minerals, amino acids and multivitamins. It has anti-influenza, anti-neuritis, promoting children's development, anti-cancer and other pharmacological functions [3]. In recent years, the growth area of Floccularia luteo-virens in alpine meadow has been decreasing at a high rate [4], and as a consequence, Floccularia luteo-virens becomes increasingly less available. Knowledge of the characteristics of SSR markers in Floccularia luteo-virens helps to mitigate the potential damage to Floccularia luteo-virens and protect biodiversity.

\section{Materials and Methods}

\section{Fungal Materials}

The fruiting bodies of Floccularia luteo-virens in the juvenile or adult stage were collected from their natural environment in
Haiyan County, Qinghai Province, China, in July, 2015. The juvenile stage is defined as the stage in which the cap diameter of Floccularia luteo-virens is $\leqq 5 \mathrm{~cm}$; while the adult stage is defined as the stage in which the cap diameter of Floccularia luteo-virens is $>5 \mathrm{~cm}$.

\section{Extraction of Total RNA}

Fresh tissues $(50 \mathrm{mg}$ ) were obtained from the cap and stipe of the fruiting bodies using a single blade for the extraction of total RNA following the manufacturer's instructions.

\section{Determination of The Purity and Concentration of RNA}

The purity and concentration of total RNA were determined using a nucleic acid and protein analyzer. The contamination of genomic DNA was determined by $1 \%$ agarose gel electrophoresis.

\section{RNA-Seq}

The total RNA samples obtained from the fruiting bodies of Floccularia luteo-virens in the juvenile or adult stage were sent to Sangon Biotech (Shanghai, China) for transcriptome sequencing. RNA-seq was performed using the Solexa Hiseq 2500 highthroughput sequencing platform (Illumina, USA) by the PE125 strategy. The sequences of the two samples were merged and de novo assembled. Repeated sequences were removed, and the sequences of $>200 \mathrm{bp}$ were selected. The longest transcript of each locus was used for Unigene. The identification and localization of 
SSRs were performed using MISA. The density of different motifs and repeat number in Transcript and Unigene were determined. The primers of SSR-PCR were designed by Primer 3 .

\section{Results}

A total of 38, 988 SSR sequences were identified from 66, 786, 044 bp sequences in the transcript. We identified 4, 715 SSRs, and 3, 741 (78.77\%) sequences contained one SSR, 675 (14.32\%) sequences contained more than one SSRs, and 309 (6.55\%) sequences contained SSRs in compound formation. In addition, a total of 22, 122 SSR sequences were identified from 23, 908, $127 \mathrm{bp}$ sequences in Unigene. We identified 2, 910SSRs, and 2, 225 (76.46\%) sequences contained one SSR, 475 (16.32\%) sequences containing more than one SSRs, and 260 (8.93\%) sequences contained SSRs in compound formation. For Transcript, the frequency of SSR motifs followed an order of mononucleotides $(37.81 \%)>$ dinucleotides $(31.48 \%)>$ trinucleotides $(23.49 \%)>$ tetranucleotides $(0.66 \%)>$ hexanucleotides $(0.50 \%)>$ pentanucleotides $(0.07 \%)$. Importantly, mononucleotides, dinucleotides, and trinucleotides accounted for about $92.78 \%$ of SSR motifs in Floccularia luteo-virens; while tetranucleotides, pentanucleotides, and hexanucleotides accounted for only $1.23 \%$.

For Unigene, the frequency of SSR motifs followed an order of dinucleotides $(42.23 \%)>$ mononucleotides $(26.00 \%)>$ trinucleotides $(22.79 \%)>$ hexanucleotides $(8.45 \%)>$ tetranucleotides $(0.57 \%)>$ pentanucleotides $(0.23 \%)$. Again, mononucleotides, dinucleotides, and trinucleotides accounted for the majority of SSR motifs in Floccularia luteo-virens. From a statistical perspective, mononucleotides had the highest frequency in Transcript, while dinucleotides had the highest frequency in Unigene. There were at least five repeats in Transcript of Floccularia luteo-virens. The motifs with 10 repeats were the most abundant $(26.36 \%)$ in Transcript, followed by that with six repeats (20.08\%), five repeats (16.37\%), seven repeats $(11.45 \%)$, eight repeats $(7.19 \%)$, and nine repeats $(4.2 \%)$, respectively. The SSRs with $\leq 11$ repeats accounted for $94.28 \%$ of the total SSRs. Furthermore, the motifs with six repeats were the most abundant $(22.71 \% \%)$ in Unigene, followed by that with ten repeats $(20.14 \%)$, five repeats $(16.43 \%)$, seven repeats $(14.88 \%)$, eight repeats $(8.83 \%)$, and nine repeats $(6.25 \%)$, respectively.

The SSRs with $\leq 11$ repeats accounted for $96.73 \%$ of the total SSRs. For Transcript, the SSRs found in this study could be categorized by 100 types of motifs (without considering sequence complementary). In general, SSRs were unevenly distributed across motif types. Motif $\mathrm{A}$ had the highest frequency of $17.50 \%$ in mononucleotides, followed by motif GT (7.83\%) in dinucleotides and motif CAA (1.53\%) in trinucleotides respectively, and the ratio of mononucleotides was $\mathrm{A}>\mathrm{T}>\mathrm{G}>\mathrm{C}$. But for Unigene, the SSRs found in this study could be categorized by 98 types of motifs. In general, SSRs were also unevenly distributed across motif types. Motif A had the highest frequency of $11.65 \%$ in mononucleotides, followed by motif TG in dinucleotides (10.86\%) and motif TGT $(1.37 \%)$ in trinucleotides, and the ratio of dinucleotides was $\mathrm{TG} / \mathrm{GT}>\mathrm{AC} /$ $\mathrm{CA}>\mathrm{AT} / \mathrm{TA}>\mathrm{AG} / \mathrm{GA}$ (considering sequence complementary).

\section{Discussion}

SSR technology is one of the most widely used molecular markers, it has been widely used in all kinds of organisms. However, there have been few studies on the development and application of SSR markers in Floccularia luteo-virens. Thus, our study may provide some insights into the application of SSR molecular markers in Floccularia luteo-virens.

In this study, we found that dinucleotide repeats were the most abundant SSR type in Unigene of Floccularia luteo-virens, which was same as that in Actinidia[5]. Moreover, dinucleotide repeats have been reported as the SSR type in ESTs with highest abundance of many animal species, such as Xiphophorus, Fundulus and others[6], and trinucleotide repeats have been reported as the SSR type with highest abundance in plants[7,8].

\section{Acknowledgement}

This project was supported by the NSFC (National Science Foundation of China) project (No.31560028), the college students of science and technology innovation fund of Qinghai University (2017-QX-24) and the excellent course in microbiology of Qinghai University (No.FL17001).

\section{References}

1. Peng JH, Lapitan NL (2005) Characterization of EST-derived microsatellites in the wheat genome and development of eSSR markers. Funct Integr Genomics 5(2): 80-96.

2. Cheng J, Zhao, Li B, Qin C, Wu Z, et al. (2016) A comprehensive characterization of simple sequence repeats in pepper genomes provides valuable resources for marker development in Capsicum. Scientific Reports 7(6): 18919.

3. Chen Q Diao Z, Han Y (2011) The Economic Value and Sustainable Utilization of Armillaria luteo-virens. World Notes on Antibiotics 32(4): 161-173.

4. Xing R, Gao QB, Zhang FQ Li Y, Ful P, et al. (2014) Isolation and characterization of 27 polymorphic microsatellite markers in Armillaria luteo-virens (Physalacriaceae). Acta Microbiologica Sinica 54(9): 10451052.

5. Fraser LG, Harvey CF, Crowhurst RN, De Silva HN (2004) EST-derived microsatellites from Actinidia species and their potential for Mapping. Theoretical and Applied Genetics 108(6): 1010-1016.

6. Ju Z, W Melissa C, Martinez A, Hazlewood L, Walter R (2005) An silico moning for simple sequence repeats from expressed sequence tags of zebrafish, medaka, Fundulus, and Xiphophorus. In Silico Biol 5: 439-463.

7. Varshney RK, Graner A, Sorrells ME (2005) Genic microsatellite markers in plants: features and applications. Trends in Biotechnology 23(1): 4855.

8. Nicot N, Chiquet V, Gandon B, Amilhay L, Legeai E, et al. (2004) Study of simple sequence repeat (SSR) markers from wheat expressed sequence tags (ESTs). Theoretical and Applied Genetics 109(4): 800-805. 
(c) (i) This work is licensed under Creative Submission Link: http://biomedres.us/submit-manuscript.php

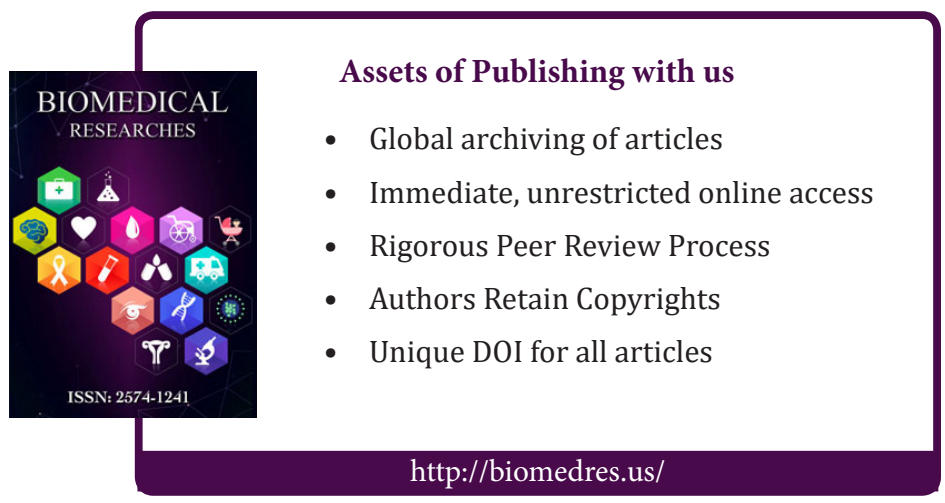

